\title{
Emergency Preparedness in Ambulatory Surgery Centers and Office-Based Anesthesia Practices
}

\author{
Shaina Drummond and Michael O'Rourke
}

\section{Emergency Preparedness in Ambulatory Surgery Centers and Office Based Anesthesia Practices}

In the past ten years, natural disasters and other emergencies have highlighted the shortcomings of healthcare systems to protect patients and healthcare team members during emergencies [1-3]. Ambulatory Surgery Centers (ASC) and Office Based Anesthesia Practices are unique healthcare environments that necessitate specific emergency planning for disasters and other emergencies. There is currently wide variability in emergency preparedness amongst Ambulatory Surgery Centers and Office Based Anesthesia Practices (OBAs). The Centers for Medicare \& Medicaid Services (CMS) recently estimated that of 5,485 ASCs in the United States, 1,414 were accredited by some regulatory agency while 4,071 ASCs were non-accredited [4]. There is no standard for emergency preparedness in non-accredited practices. Even those ASCs with accreditation may have different levels of emergency preparedness as accreditation organizations can have different standards or no standards at all for emergency preparedness. It is important that healthcare providers at all ambulatory surgery centers and office based anesthesia practices are aware of existing regulations for emergency preparedness and work to ensure patients and staff as well as physical resources are protected during natural disasters and other emergencies.

\footnotetext{
S. Drummond $(\bowtie)$

Department of Anesthesiology and Pain Management, UT Southwestern Medical Center, Dallas, TX, USA

Parkland Ambulatory Surgery Center, Dallas, TX, USA

e-mail: shaina.drummond@utsouthwestern.edu

\section{O'Rourke}

Department of Anesthesiology and Perioperative Care, Loyola University Medical Center, Maywood, IL, USA

e-mail: miorourke@lumc.edu
} 


\section{Types of Emergencies}

Emergencies and natural disasters stress a healthcare system and lead to challenges in protecting patients' and providers' welfare [2, 3, 5, 6]. Hospitals and other healthcare systems need to protect patients, employees, and physical resources during such events while continuing to provide care to those in need. Natural disasters can include earthquakes, tornadoes, hurricanes, floods, or tsunamis. Natural disasters generally affect a large number of people and can require coordination of both local and national resources to respond to healthcare needs [1]. The earthquake and subsequent tsunami that struck Japan in 2011 as well as Hurricane Katrina and the subsequent flooding in Louisiana in 2005 are examples of the widespread destruction that can be caused by natural disasters. In both of these cases, healthcare systems were disrupted and largescale resources were needed to respond to the subsequent healthcare emergencies [7, 8].

The dialysis community provides perspective on the importance of emergency preparedness. In response to an earthquake in Armenia in 1998 that disrupted dialysis services in the country, the International Society of Nephrology formed the Renal Disaster Relief Task Force. This task force provides expertise or resources to communities dealing with natural disasters. In response to an earthquake in Haiti in 2010, the organization provided 25 volunteers over a 2 month period to treat patients with both acute and chronic kidney injury [3]. Despite major organizational difficulties in Haiti, the task force organized renal care for patients in Haiti and arranged transfer of patients to the neighboring Dominican Republic when needed. The experience in Haiti highlights the efforts of an outside organization to organize dialysis care when the existing resources are not sufficient to adequately respond to a natural disaster. A major earthquake also struck the country of Chile in 2010 near the country's second largest city of Concepción. The earthquake disrupted dialysis care for 2500 people [3]. In Chile, local nephrologists were able to coordinate patient care including transfer of patients and adjustment in dialysis schedules. As a result, $100 \%$ of the affected patients had access to dialysis six days after the earthquake despite less than $60 \%$ of dialysis centers being operational [3]. In this case the Renal Disaster Relief Task Force served in an advisory role to the local nephrologists organizing care [3]. While it is difficult to compare earthquakes or other natural disasters, it is clear that having an emergency plan and resources in place prior to an emergency aids in patient care during an emergency.

Natural disasters are prototypical emergencies which can disrupt healthcare systems. However, numerous other emergencies exist. Infectious disease emergencies can be local or global in nature. Recent well publicized outbreaks of the Avian influenza, SARS and the Ebola virus are examples of infectious disease emergencies with global implications. However, a local outbreak of influenza could also require a response from an ambulatory surgery center. Acts of terrorism including chemical, biological, radiological, nuclear, or explosive attacks are a separate class of emergency. Active shooter events in the United States have prompted a greater awareness of these events and the need to create an emergency plan for such events. Local events that could disrupt ambulatory surgery centers or office based 
anesthesia practices include severe weather or the loss of utilities or other services at the practice site. In addition to all these emergencies, surgery specific emergencies like local anesthetic systemic toxicity events or onset of malignant hyperthermia are patient emergencies requiring rapid responses. Due to the large and varied types of emergencies that can affect an ambulatory surgery center or office based anesthesia practice, it is important to assess a facility's need and ability to respond to various emergencies.

\section{Risk Assessment}

The first step in emergency planning is performing a risk assessment for the particular practice. An "all hazards" approach is the preferred approach for risk assessment. The "all hazards" approach is used by a variety of organizations including the World Health Organization and the United States Federal Emergency Management Agency (FEMA) [9-11]. It is also the approach recommended by The Joint Commission for healthcare organizations in emergency planning [12].

In an "all hazards" approach the practice attempts to identify all emergencies which could occur at the facility and in the community in which the facility is located. This process should include consideration of the general geographical area and the practice's specific location in the community. It should also consider the patient and staff population typically present at the facility including consideration for children, the elderly, or patients with disabilities [4]. Once all possible emergencies are identified they are evaluated for the likelihood that they would occur. To use recent natural disasters as an example, it would be appropriate for an ambulatory surgery center in coastal Louisiana to prioritize planning for a hurricane or flooding whereas a facility in Los Angeles might more appropriately plan for an earthquake. In addition to natural disasters, emergencies that every ambulatory surgery center or office based anesthesia practice should assess are severe weather, active shooter in the facility or nearby area, loss of utilities at the practice, and medical emergencies like malignant hyperthermia, local anesthetic systemic toxicity, or an unresponsive patient.

\section{Development of an Emergency Plan}

Once a risk assessment has been completed, an individualized emergency plan for the practice should be developed. To be meaningful and work effectively, the emergency plan must be individualized for the particular Ambulatory Surgery Center or Office Based Anesthesia practice and take into account the resources, staff, and patient population of a particular practice. Once developed, the emergency plan should be reviewed and updated annually.

Certain portions of an emergency plan may be very specific. For instance, if an office based anesthesia practice provided service for patients receiving local anesthetic injections as part of cosmetic or reconstructive procedures, it would be 
recommended the practice have a specific plan for managing a patient with local anesthetic systemic toxicity (LAST). The emergency plan would be tailored to the particular resources and staff at the practice. This could include having assigned roles for staff in a LAST event such as bringing emergency airway equipment to the patient bedside, bringing lipid emulsion therapy to the bedside, or assisting with airway management. The plan could also ensure availability of lipid emulsion therapy and the American Society of Regional Anesthesia and Pain Medicine's Checklist for the Treatment of Local Anesthetic Systemic Toxicity for any such event [13]. The plan could include annual LAST drills with staff so resources and roles are well established prior to such an event. By having a specific plan for a specific LAST emergency identified in its risk assessment, an office based anesthesia practice might better provide timely and appropriate care to its patients during such an emergency.

The emergency plan should include a framework for managing larger or previously unidentified emergencies. This could include identification of leadership roles during the emergency, a plan for sheltering or evacuating patients and their families, a plan for sheltering or evacuating staff, and a plan for preserving physical resources at the practice. Large or unexpected emergencies are unpredictable in nature, but establishing a management plan for such events as part of a larger emergency plan provides a baseline level of expectations and responsibilities amongst staff for handling such events. Establishing this plan prior to the event allows for staff to know their role during an emergency. If a large or unexpected emergency event arises, leadership can adapt the plan as needed to that specific emergency.

\section{Key Elements of an Emergency Plan}

Though emergency plans will differ depending on the type of practice, there are several key elements which should be included. CMS provided an outline of such key elements in its final rule on Emergency Preparedness Requirements for Medicare and Medicaid Participating Providers and Suppliers in 2016 [4]. CMS identified three essential requirements for maintaining healthcare services during an emergency: safeguarding human resources, maintaining business operations, and protecting physical resources [4]. CMS requires that a facility's emergency plan include a list of services it is able to provide during an emergency [4]. Obviously, most ambulatory surgery centers and office based anesthesia practices that provide elective surgical care would not continue to provide elective care during a true emergency. However, CMS requires that the practice consider what services would or could be provided during an emergency [4]. CMS also requires the practice to attempt to collaborate with local, tribal, regional, state, or federal emergency officials [4]. In addition, an emergency plan should address the continuity of operations, including the delegation of authority and succession plan [4].

An emergency plan also needs to account for care of staff and patients during an emergency. An emergency plan should include a system to track the location of 
staff and sheltered patients in an ambulatory surgery center during an emergency $[4,12]$. A means to shelter in place for patients, staff, and volunteers should also be included.

When sheltering in place is not feasible, an emergency may require the evacuation of a facility. Hence, a safe evacuation plan is a necessary component of any emergency plan. The evacuation plan should consider the abilities of staff, patients, families, and volunteers typically present at the practice. The plan should include planning for care and treatment of evacuees both during and after evacuation. Means of transportation and evacuation locations should be documented in an evacuation plan. If on-duty staff or patients are transferred during an emergency, documentation of the receiving facility's name and location should be made at the ambulatory surgery center [4, 12]. Finally, a primary means of communication amongst staff and patients should be established as well as alternate means if the primary method is disrupted during the emergency.

Medical documentation is important at all times including during an emergency. Hence, an emergency plan should include a method to preserve patient information and protect patient confidentiality during an emergency. For example, during loss of utilities a facility should have a plan to maintain medical documentation and ensure those records are secure and available for patients and staff.

\section{Assessing Emergency Needs and Equipment}

Planning for an emergency necessitates the creation of emergency preparedness checklists to address several key requirements: 1) Patient notification systems (in case of emergency or closure); 2) Staff notification system (in case of emergency or closure); 3 ) Medical records backup plan (e.g., cloud servers for electronic records, short term use of paper records); 4) Remote medical record access, 5) Billing services (continuity plan); 6) Security at the facility; 7) Utility emergency shut-off location and procedures; 8) Service availability (define available priority services); 9) Disaster planning for staff (who is expected to work and when) [5].

In times of crisis, staffing at an ASC can become a major issue. It is important to identify a variety of staffing strategies before an emergency to ensure that proper staffing levels can be maintained as staff may have difficulty reporting for work because of the incident [5]. A triage plan should be developed for use in time of emergency. For example, utilizing all members of the team, from the physician and nurses to the medical assistants and housekeeping staff to serve on teams in specific areas to better facilitate patient care. It is important for everyone available to play a role on a team.

If an ASC is affiliated with a larger hospital entity, it is possible that the ASC could be used to treat less acute surgical patients during an emergency situation if the main hospital is at capacity due to a mass casualty. Code carts and medications should be fully stocked, organized and maintained. In addition, emergency supplies (e.g., emergency airway kits, fiberoptic scopes, glidescopes, backup anesthesia machines, full oxygen tanks, extra batteries) and medications (e.g., intralipid 
emulsion if applicable and dantrolene for treatment of malignant hyperthermia) should be kept up-to-date and checked regularly to ensure properly functioning equipment. It is also important for ASC leadership to know the location of suppliers and whom to contact if more supplies are needed. In the event that an ASC is in close proximity to a larger affiliated hospital system, a courier service between both entities for laboratory and equipment needs could be utilized.

The outbreak of the Ebola virus in Dallas, TX highlighted the importance of infection control planning in emergency preparedness. ASCs should have a staff person whose designated responsibility is infection control. Even though most ASCs are unlikely to face a largescale outbreak of an infectious disease, it is still important for ASCs to have procedures in place for the proper collection, holding, and disposal of biological waste and to establish systems in case disposal measures are disrupted. There should also be procedures in place for the isolation of a patient who is discovered to have an infectious disease along with procedures for the safe transfer of a patient to an appropriate facility [14]. Most ASCs do not electively take care of patients with certain types of communicable diseases due to their lack of isolation facilities.

\section{Communication}

Communication is a critical component of any emergency operations plan. CMS requires facilities to have a written emergency communication plan that describes how the facility will coordinate continued patient care within the facility, with outside healthcare providers, and with state/local public health departments in the case of an emergency. Location specific considerations must also be incorporated, such as limited access to the internet or phone capabilities for those practicing in more rural locations. During an emergency situation, it is important for an ASC to have established methods to communicate with partner facilities and local emergency responders should there be a loss of landline and cell phone capabilities. Components of effective communication in a time of emergency include: 1) a functional audible paging system to announce clear text messages to alert staff, patients and visitors of the emergency; 2) procedures for calling staff back to the organization, when needed and also a process whereby staff report if they become aware of an emergency and landlines or cell phones cannot be used to contact staff; ASCs should have a phone tree or another method of rapidly communicating with all their staff in case of an emergency. 3) a method to communicate with patients to cancel procedures when necessary [14].

Administration and the governing body of the ASC are responsible for the development and approval of the disaster preparedness plan. The ASC should designate and clearly communicate with their staff, the person responsible for the emergency operations plan. This designated person should have this responsibility listed in their job description. Other examples of responsibilities that should also be included in the job description include: 1) maintain current knowledge of changes to environment of care and emergency management standards; 2) establish and implement safety and emergency preparedness policies appropriate for the ASC; 3) coordinate emergency 
preparedness plan with partner organizations and other emergency response partners; 4) ensure safe environment for patients, visitors, and employees; 5) evaluate safety and emergency preparedness orientation and annual education and training programs for employees and ensure compliance with all applicable standards including Centers for Medicare and Medicaid (CMS), Joint Commission (or other accrediting agency); 6) recommend and monitor trends for improvement opportunities related to employee, visitor, and patient incidents; 7) plan, implement and evaluate environmental rounds; 8) monitor and evaluate timely and thorough completion of Emergency Preparedness exercises and complete the After Action Report (AAR) for all exercises, including identification of Corrective Actions; 9) evaluate and up-date the Safety and Emergency Preparedness Programs annually [14].

Physicians working in the ED the night of the recent Las Vegas mass casualty shooting revealed that many lives were saved due to the ability of physician leaders and nurses to effectively organize patients into clear physical zones by communicating clearly with their staff to avoid wasting time that could be better spent on resuscitating patients. In any emergency situation, effective communication is key to saving lives. One effective communication method is the SBAR mnemonic: $\underline{\text { Situation, }}$

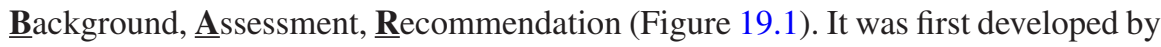
the military then adapted to the aviation industry to combat communication barriers
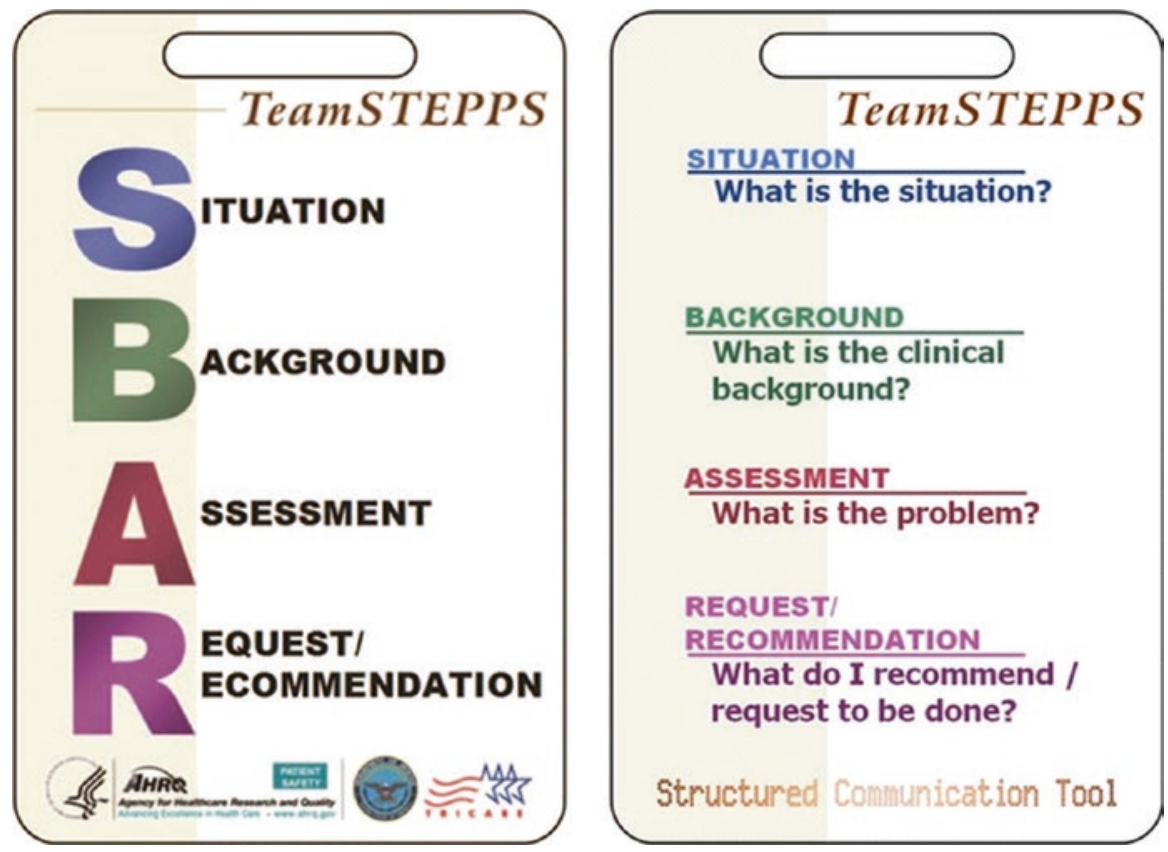

Figure 19.1 "SBAR" TeamSTEPPS ${ }^{\circledR}$ 2.0 Pocket Guide. Team Strategies \& Tools to Enhance Performance \& Patient Safety (TeamSTEPPS $\left.{ }^{\circledR}\right)$ 2.0. Agency for Healthcare Research and Quality, Rockville, Maryland (USA). December 2013. http://www.ahrq.gov/teamstepps/instructor/essentials/pocketguide.html. (Reprinted with permission of the United States Agency for Healthcare Research and Quality.) 
inherent in the work environment, which were causing life threatening crashes. In 2002, it was introduced into the healthcare system by Kaiser Permanente after the realization that there are fundamental similarities between healthcare and aviation/ military environments--both are high stress, high risk, time sensitive environments, where wrong decisions can be life threatening. It was initially introduced into rapid response teams with the goal of improving patient safety by having a standardized process for early intervention before a patient's condition deteriorates. The goal of SBAR is to overcome communication barriers by providing a standard concise framework for conveying pertinent information between members of the healthcare team, which is essential for fostering a culture of patient safety [15].

\section{Training and Testing}

Centers for Medicare and Medicaid have mandated that ASCs take part in two annual emergency preparedness tests to assess an emergency plan's effectiveness. The first exercise should be a community based drill, if available. If this is unavailable, then the ASC is required to conduct an individual facility-based drill. For the second exercise, an ASC would be required to conduct either a facility based drill or a tabletop exercise. While the drill does not have to test the response to every identified hazard, it is expected to test a significant portion of the plan. Drills and exercises should be conducted to practice policies and procedures, evaluate the effectiveness of the emergency plan, and identify areas of improvement. In addition, it is important for an ASC to maintain documentation of all emergency training completed by staff $[4,14,16]$.

Implementation of emergency organized drills and testing is a complex task requiring significant resources in terms of cost, manpower, training, and time commitment. Given the fact that the process of preparing healthcare professionals to manage emergencies is both complicated and costly, it is important to optimize the emergency preparedness program by investing resources in the common components that may improve preparedness for different emergency scenarios. This is where simulation based drills could play a very important role. Simulation based training can increase emergency preparedness by exposing healthcare staff to real world emergency situations. Benefits of team based simulation include assessment of the current internal emergency disaster plans and the team's readiness to deal with the situation at hand, development and application of clinical and critical thinking skills, promotion of team based collaboration and communication skills, discovery of flawed processes, and problem solving through corrective action plans [17].

\section{Development of a Corrective Action Plan}

While drills and testing are necessary components of disaster planning, it is equally important that team members debrief at the end of an exercise by creating an after action report. The report should identify and document deficiencies 
and recommendations for improvement otherwise known as "corrective actions". A corrective action plan (CAP) includes: 1) the purpose of the drill or exercise, 2) participating agencies 3) scenario overview, 4) exercise objectives (e.g., communications, resource mobilization), 5) areas for improvement. The plan should also include a full description of the deficiency; the action that should be taken; the resources required to address the deficiency; and justification for the need to correct the deficiency. Prioritization of corrective actions is helpful because funding and time are usually limited [18]. Prioritization can also identify significant deficiencies that should be reported to management and corrected as quickly as possible. Criteria or categories for corrective action may include the following: 1) hazards to health and safety, 2) regulatory compliance, 3) hazards to property, operations, the environment or the entity 4) conformity to national standards. Action on deficiencies should be assigned to the person or department best able to address the issue. A due date should be assigned and the corrective action database reviewed regularly to track progress. If there are changes in processes or practices as a result of the corrective action plan, they should always be clearly communicated to staff, physicians, and governing bodies prior to implementation [17-19].

\section{Recovery}

Following an emergency situation that results in major disruption of services or evacuation, recovery measures are required to restore the facility to baseline level of operations. Major facility disruptions are required to be reported to the state Department of Health (DOH) and patient care activities may only resume after an inspection by the $\mathrm{DOH}$.

\section{Conclusion}

Over the past decade, the need to respond to emergencies, natural disasters, and mass casualty events has been highlighted in the United States and throughout the world. Ambulatory Surgery Centers and Office Based Anesthesia Practices are unique healthcare environments that necessitate specific emergency planning for disasters and other emergencies. Such practices typically do not have rapid response or code teams on site and often have leaner staffing than other parts of a health system. CMS has developed a series of guidelines to establish consistent emergency preparedness requirements for healthcare providers specifically in the ambulatory surgery setting. Components of emergency preparedness consist of planning, organizing, communicating, equipping, training, and exercising (Figure 19.2). While the development of an emergency plan is an important first step in the process of emergency preparedness, it is also important to continuously reevaluate the emergency plan through drills, simulation, and corrective action plans. Without periodic maintenance, the emergency plan will 


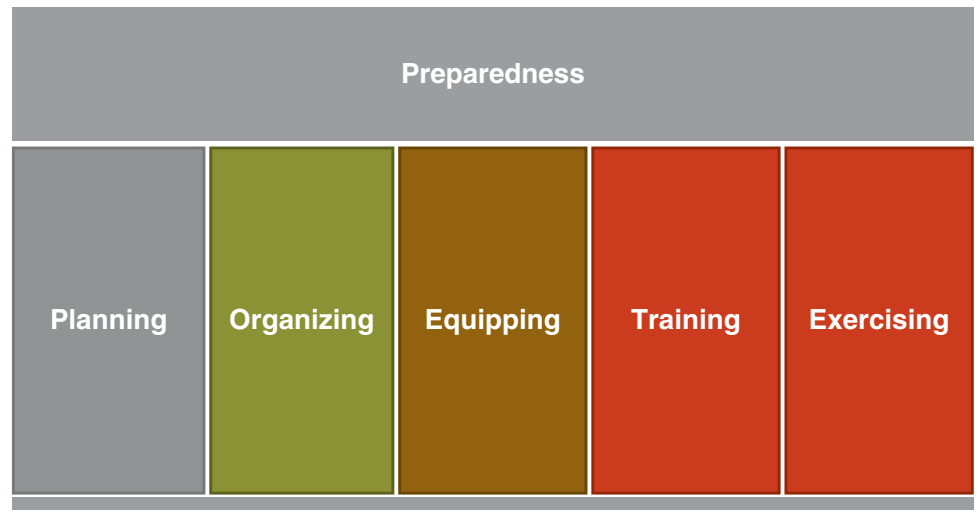

Figure 19.2 Preparedness. Courtesy of Timothy Riecker, Emergency Preparedness Solutions, LLC, Utica NY. https://triecker.wordpress.com/

become outdated and ineffective. Emergency preparedness is an ongoing process in which leadership, commitment, action, funding, and partnerships at all levels must be sustained.

\section{References}

1. Craven R. Managing anaesthetic provision for global disasters. BJA $\mathrm{Br} \mathrm{J}$ Anaesthesia. 2017;119(suppl_1):i126-34.

2. Der-Martirosian C, Pinnock L, Dobalian A. Accessing VA healthcare during large-scale natural disasters. Hosp Top. 2017;95(1):1-9.

3. Vanholder R, Borniche D, Claus S, Correa-Rotter R, Crestani R, Ferir M, et al. When the earth trembles in the Americas: the experience of Haiti and Chile 2010. Nephron Clin Pract. 2011;117(3):c184-97.

4. Department of Health and Human Services. Centers for Medicare \& Medicaid Services. Medicare and Medicaid Programs; Emergency Preparedness Requirements for Medicare and Medicaid Participating Providers and Supplier. Fed Regist. 2016;81(180):63860-4024.

5. Haeri S, Marcozzi D. Emergency Preparedness in Obstetrics. Obstet Gynecol. 2015;125(4):959-70.

6. Miller A, Arquilla B. Chronic diseases and natural hazards: impact of disasters on diabetic, renal, and cardiac patients. Prehosp Disaster Med. 2008;23(02):185-94.

7. Gray N, Wolley M, Liew A, Nakayama M. Natural disasters and dialysis care in the AsiaPacific. Nephrology. 2015;20(12):873-80.

8. Dent L, Finne K, Lurie N. Progress in emergency preparedness for dialysis care 10 years after Hurricane Katrina. Am J Kidney Dis. 2015;66(5):742-4.

9. Adini B, Goldberg A, Cohen R, Laor D, Bar-Dayan Y. Evidence-based support for the allhazards approach to emergency preparedness. Israel J Health Policy Res. 2012;1(1):40.

10. Emergency Management Institute (EMI) | All Hazards Position Specific Webiste [Internet]. Training.fema.gov. 2017 [cited November 2017]. Available from: https://training.fema.gov/ allhazards/.

11. Federal Emergency Management Agency. Comprehensive Preparedness Guide 101: developing and maintaining emergency operations plans. 2010. p. 2-2 to 3-5.

12. Joint Commission on Accreditation of Healthcare Organizations. Standing together: an emergency planning guide for America's communities. 2005. p. 15-7. 
13. Checklist for Treatment of Local Anesthetic Systemic Toxicity [Internet]. Asra.com. 2012 [cited November 2017]. Available from: https://www.asra.com/advisory-guidelines/article/3/ checklist-fortreatment-of-local-anesthetic-systemic-toxicity.

14. Centers for Medicare \& Medicaid Services. Guidelines for Disaster Preparedness for Ambulatory Surgery Centers. Available from: http://www.michiganasc.com/images/ Guidelines_for_Disaster_Preparedness_for_ASC.pdf. p. 4-12.

15. Pope B, Rodzen L, Spross G. Raising the SBAR: How better communication improves patient outcomes. Nursing. 2008;38(3):41-3.

16. Ambulatory Surgery Center Association. Emergency Preparedness. September 2016. Available from: https://www.ascassociation.org/federalregulations/emergency-preparedness.

17. Vaidya A. 5 key elements of emergency preparedness in ASCs. Becker's Hospital Review. Webinar: https://www.beckersasc.com/asc-turnarounds-ideas-to-improve-performance/5-keyelements-of-emergency-preparedness-in-ascs.html. May 11, 2015. Linked to Slides by Deihs L, and Kilgore, K. Is your ASC ready for a crisis? Simulation-based training in emergency management. Accreditation Association for Ambulatory Health Care, Inc. May 5, 2015.

18. Department of Homeland Security. Corrective Action. Available from redy.gov: https://www. ready.gov/business/program/corrective.

19. Centers for Medicare \& Medicaid Services. Corrective Action Plan (CAP) Process. Available from https://www.cms.gov/research-statistics-data-and-systems/monitoring-programs/perm/ downloads/2013correctiveactionpowerpoint.pdf. 